\title{
The effect of aging on the autophagic and heat shock response in human peripheral blood mononuclear cells
}

\author{
JJ McCormick ${ }^{1}$, TA VanDusseldorp ${ }^{1,2}$, CG Ulrich ${ }^{1}$, RL Lanphere ${ }^{3}$, K Dokladny ${ }^{4}$, \\ PL Mosely ${ }^{5}$, CM Mermier ${ }^{1}$

\footnotetext{
${ }^{1}$ Department of Health, Exercise, and Sports Sciences, University of New Mexico, Albuquerque, NM, USA

${ }^{2}$ Department of Exercise Science and Sport Management, Kennesaw State University, Kennesaw, GA, USA

${ }^{3}$ Department of Kinesiology \& Health Promotion, University of Kentucky, Lexington, KY, USA

${ }^{4}$ Department of Internal Medicine, University of New Mexico, Albuquerque, NM, USA

${ }^{5}$ Departments of Medicine and Biomedical Informatics, University of Arkansas for Medical Sciences,
} Little Rock, AR, USA

Received: July 19, 2017

Accepted: August 7, 2018

\begin{abstract}
Autophagy is a lysosome degradation pathway through which damaged organelles and macromolecules are degraded within the cell. A decrease in activity of the autophagic process has been linked to several age-associated pathologies, including triglyceride accumulation, mitochondrial dysfunction, muscle degeneration, and cardiac malfunction. Here, we examined the differences in the autophagic response using autophagy-inducer rapamycin (Rapa) in peripheral blood mononuclear cells (PBMCs) from young (21.8 \pm 1.9 years) and old (64.0 \pm 3.7 years) individuals. Furthermore, we tested the interplay between the heat shock response and autophagy systems. Our results showed a significant increase in LC3-II protein expression in response to Rapa treatment in young but not in old individuals. This was associated with a decreased response in MAP1LC3B mRNA levels, but not SQSTM1/p62. Furthermore, HSPA1A mRNA was upregulated only in young individuals, despite no differences in HSP70 protein expression. The combined findings suggest a suppressed autophagic response following Rapa treatment in older individuals.

Keywords: autophagy, aging, HSP, PBMC, rapamycin
\end{abstract}

\section{Introduction}

Autophagy (literally translated as "self-eating") is a lysosome degradation pathway through which damaged organelles and macromolecules are degraded within the cell $(28,29)$. The autophagic process plays a homeostatic role in cells that is crucial for the metabolic balance between the synthesis, degradation, and subsequent turnover of cytoplasmic materials in a stressful environment (8). Autophagy is classified into three types that can be distinguished based on the cellular components that are sequestered and degraded consisting of: microautophagy, chaperone-mediated autophagy, and macroautophagy (herein referred to as autophagy) (39).

Autophagic flux occurs in several main steps including the sequestration of cargo into the autophagosome, the delivery of cargo to lysosomes, and its subsequent breakdown and release of macromolecules back into the cytosol (23). Microtubule-associated protein light

Corresponding author: James J. McCormick

Department of Health, Exercise, and Sport Science, University of New Mexico

Johnson Center B143 Albuquerque, NM 87110, USA

Phone: +505 350 8370; Fax: +505 277 2657; E-mail: aeneid@unm.edu 
chain 3 (LC3) is the most commonly used protein to examine autophagy, through which the conversion of the unconjugated cytosolic form of LC3-I to the phosphatidylethanolamineconjugated form of LC3-II targets the autophagosomal membrane resulting in formation of the autophagolysosome and its subsequent degradation $(20,23,38)$. Sequesterome 1/p62 (SQSTM1/p62) tags and binds molecules for transportation to the autophagosome, which is then degraded by the autophagolysosome. Induction of autophagy is indicated by increases in LC3-II and its concomitant degradation or the degradation of SQSTM1/p62 (23).

A decrease in activity of the autophagic process is a shared characteristic of nearly all aged organisms, which may have a causative role in the functional deterioration of biological systems during aging (43). Over the past decade, accumulating evidence supports a direct role for autophagy in the aging process $(2,3,17,25,44)$. Autophagy-related proteins (ATGA) and other proteins (such as Sestrin 1) required for autophagy induction have been shown to have reduced expression in aged tissues. For instance, normal aging of the human brain is associated with downregulation of Atg5, Atg7, and Beclin 1 (31), whereas deficiencies in Atg 1, Atg 8, and Sestrin 1 are associated with decreased lifespan, suggesting an enfeeblement of the autophagic response that may contribute to the aging phenotype $(26,42)$. Furthermore, downregulation of proteins required for autophagic functioning has been linked to age-associated pathologies, including triglyceride accumulation, mitochondrial dysfunction, muscle degeneration, and cardiac malfunction $(26,41)$. In addition, the genetic and agerelated loss of autophagic functioning has been linked to the development of several metabolic and neurodegenerative diseases, such as diabetes, Huntington's disease, Alzheimer's disease, and Parkinson's disease (25).

Few studies have tested the effect of correcting age-associated autophagic deficits on aging-related phenotypes. Melendez et al. (34) observed that inhibition of the insulin-like growth factor (IGF) pathways cause an increase in autophagy in Caenorhabditis elegans, thus increasing longevity; however, inhibition of autophagy through mutation of essential ATG genes prevents longevity gains. Caloric restriction (CR) or reduced food intake without malnutrition is the strongest known physiological inducer of autophagy (29). CR extends the life span of most tested animals, in which it reduces incidence of diabetes, cardiovascular disease, cancer, and brain atrophy (6). Autophagic induction from CR causes activation of both the AMPK or Sirtuin 1 energy sensors and engages in a positive forward loop of mutual activation (4). Moreover, CR-initiated inhibition of IGF results in the downregulation of mammalian target of rapamycin (mTOR), causing an increase in the autophagic flux (21).

Recently, heat shock proteins (HSPs) have been found to have a regulatory role in the autophagic response (11). HSPs are molecular chaperones that assist in correct folding of newly synthesized proteins, and in the translocation, degradation, and reactivation of damaged proteins (10). HSP70, a member of the HSP family of proteins, offers protection against damaging factors at the level of the macromolecules, single cells, and whole organisms (11). Overexpression of HSP70 has an inhibitory role on autophagy that may be modulated through the mTOR/Pkb (protein kinase B, also known as Akt) pathway (11); however, the regulatory role of HSP70 on rapamycin (Rapa)-induced autophagy remains unknown in aged individuals.

The purpose of this study was to examine differences in basal and Rapa-induced autophagy in the peripheral blood mononuclear cells (PBMCs) of young and old individuals. In addition, we aimed to examine the interplay between HSP70 and Rapa-induced autophagy as it relates to aging. 


\section{Materials and Methods}

\section{Subjects}

The study was approved by the university's Human Research Review Committee (HRPO12-224) and all subjects provided written informed consent. Adult participants between the ages of 20-25 and 60-72 years were recruited for the study, who were free of known illness or disease. Subjects who are falling outside of the age ranges, currently taking medication known to upregulate autophagy, such as glucose lowering or statin medications, or experiencing an acute illness were excluded. Furthermore, participants were asked to refrain from exercise $24 \mathrm{~h}$ prior to their blood draw and all participants were non-smokers. A total of 10 males (5 young and 5 old) and 10 females (5 young and 5 old) were recruited to participate in this study $(n=20)$. Participants were divided into two groups by age: young $(21.8 \pm 1.9$ years $)$ and old $(64.0 \pm 3.7$ years $)$.

\section{PBMC collection and cell experiments}

Participants were asked to visit the lab on a single occasion. Venous blood was collected via venipuncture from an antecubital vein. PBMCs were isolated from whole blood suspended in histopaque (Histopaque-1077, Sigma-Aldrich, USA), counted to ensure equal cell densities between all participants (approximately $4.0 \times 10^{-6}$ cells), and incubated at $37^{\circ} \mathrm{C}$ for a period of $24 \mathrm{~h}$ in cell culture medium containing MegaCell RPMI-1640 (F4135, Sigma-Aldrich, USA), 1\% L-glutamine (Thermo Fisher Scientific, USA, 25030081), 1\% Penicillin-Streptomycin solution (Thermo Fisher Scientific, 15140122), and 5\% fetal bovine serum (F4135, SigmaAldrich, USA) to allow for cell homogenization. PBMCs were then transferred to cell culture plates and were either treated with dimethyl sulfoxide (DMSO) vehicle (472301, SigmaAldrich), bafilomycin (BAF) A1 in DMSO (InvivoGen, tlrl-bafl, USA) (100 nM), or BAF and Rapa in DMSO (InvivoGen, tlrl-rap) $(0.5 \mathrm{nM})$ for $2 \mathrm{~h}$ in $37^{\circ} \mathrm{C}$. BAF was used to block the formation of the autophagolysosome preventing LC3-II degradation, thus enabling the quantification of autophagy via LC3-II accumulation $(23,45)$. Treatment with Rapa simulated starvation-induced autophagy via upstream inhibition of mTOR. Cells were immediately harvested and stored at $-80{ }^{\circ} \mathrm{C}$ until analyzed.

\section{Immunoblot analysis}

Cells were lysed in a modified radioimmunoprecipitation buffer (Tris- $\mathrm{HCl} 8.0 \mathrm{pH}$; Invitrogen, USA, 15568-025), 0.5 M EDTA (Invitrogen, 15568-020), 1.5 M NaCl (S9888, SigmaAldrich), $1 \%$ Triton $\times 100$ (Sigma-Aldrich, $\times 100$ ), and freshly added protease (Thermo Fisher Scientific, 78430) and phosphatase (Thermo Fisher Scientific, 7842) inhibitors. HSP70, LC3, SQSTM1/p62, and $\beta$-actin were resolved by electrophoresis in a $12 \%$ polyacrylamide gel (Bio-Rad, 456-144, Hercules, CA). Proteins were transferred to cellulose membranes

(Bio-Rad, 162-0094) and then blocked in Tris-buffered saline (150 mM NaCl, pH 8.0), containing $0.2 \%$ polysorbate (Tween 20; Bio-Rad, 170-6531) detergent and 5\% powdered milk (Bio-Rad, 170-6404). Membranes were then incubated in Tris-buffered saline, containing $0.2 \%$ polysorbate detergent and $5 \%$ bovine serum albumin (A9418, Sigma-Aldrich) with primary antibodies including: LC3 (L7543, Sigma-Aldrich), SQSTM1/p62 (Abcam, USA, ab56416), HSP70 (Enzo, USA, P08107), and $\beta$-actin (A5441, Sigma-Aldrich). Primary antibodies were detected by horseradish peroxidase-labeled secondary antibody (Goat antirabbit; Cell Signaling, 7074s; Goat anti-mouse; Cell Signaling, 70076s) binding, which was 
detected using Santa Cruz Western blotting luminol reagents (Santa Cruz Biotechnology, Santa Cruz, CA) using the ChemiDoc XRS+ (Bio-Rad).

Quantitative reverse transcription-polymerase chain reaction ( $q R T-P C R$ )

Total RNA was isolated from human PBMCs using the QIAshredder (Qiagen, USA, 79654) and RNeasy Mini Kit (Qiagen, 74104) and then reverse transcribed using the cDNA Reverse Transcription Kit (Roche, 64869866001). cDNAs were amplified in a StepOnePlus Lightcycler (Grand Island, NY, USA) using the following amplification conditions: PCR initial activation step: $95^{\circ} \mathrm{C}$ for $10 \mathrm{~min}$; two-step cycling: 40 cycles of denaturation, $95^{\circ} \mathrm{C}$ for $15 \mathrm{~s}$; combined annealing/extension: $60^{\circ} \mathrm{C}$ for $1 \mathrm{~min}$. The sequence-specific primers (Table I) used in the reactions were: MAP1LC3B (Applied Biosystems, USA, Hs00792944_s1), SQSTM1/ p62 (Applied Biosystems, Hs01061917_g1), and HSPA1A (Applied Biosystems, Hs00359163_s1) that were used as previously described (1, 5). Gene expression was normalized to reference gene $\beta$-actin (internal control gene; Applied Biosystems, 4326315E). Relative quantification of the MAP1LC3B and HSPA1A genes was calculated relative to $\beta$-actin and the mean fold change in expression of the MAP1LC3B and HSPA1A genes was calculated using $2^{-\Delta \Delta \mathrm{CT}}$ method.

Image analysis and statistical methods

Adobe Photoshop (version 6, Adobe Systems Incorporated) was used to quantify immunoblot band intensity. Two-way analysis of variance (Age $\times$ Treatment) was performed followed by the Bonferroni post hoc test. Significance was determined as $p<0.05$.

\section{Results}

Effect of Rapa treatment on autophagy and HSP70 protein expression in young and old individuals

To understand the impact of Rapa-induced autophagy in aging, PBMCs from young and old individuals were incubated for $24 \mathrm{~h}$ and treated with either DMSO vehicle, BAF (100 nM), or Rapa $(0.5 \mu \mathrm{M})$ for $2 \mathrm{~h}$. Western blot was used to determine LC3-II, SQSTM1/p62, and HSP70 protein expression. Gene expression of MAP1LC3B, SQSTM1/p62, and HSPA1A was determined using qRT-PCR. All proteins and genes were normalized to $\beta$-actin, a commonly used procedure $(23,35)$. Treatment with $\mathrm{mTOR}$ inhibitor, Rapa, for $2 \mathrm{~h}$ elicited a significant increase in LC3-II protein expression in young and a non-significant increase in old individuals (Figs 1 and 2). As BAF inhibits the autophagic degradation of LC3-II, accumulation of LC3-II is indicative of an increased autophagic response (45). Thus, our findings suggest that

Table I. Forward and reverse primers used for qRT-PCR

\begin{tabular}{|l|l|l|}
\hline Gene & \multicolumn{1}{|c|}{ Forward primer } & \multicolumn{1}{c|}{ Reverse primer } \\
\hline MAP1LC3B & 5'-AGCAGCATCCAACCAAAATC-3' & 5'-CTGTGTCCGTTCACCAACAG-3' \\
\hline SQSTM1/p62 & 5'-CACCTGTCTGAGGGCTTCTC-3' & 5'-AGTTTCCTGGTGGACCCATT-3 \\
\hline HSPA1A & 5'-TGGACTGTTCTTCACTCTTGGC-3' & 5'-TCCGGAGAGTTCTGGGATTGTA-3' \\
\hline
\end{tabular}

qRT-PCR: quantitative reverse transcription-polymerase chain reaction 
A

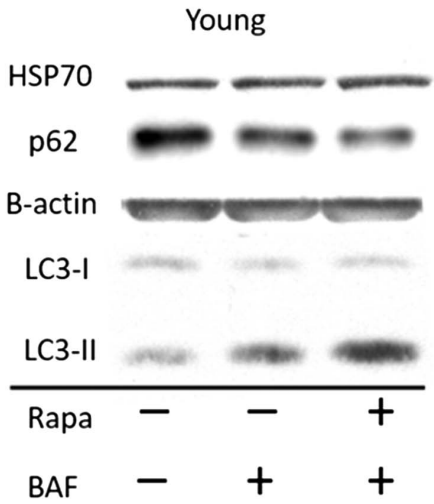

B

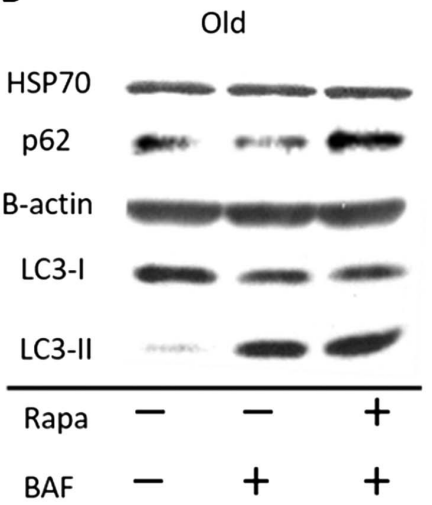

Fig. 1. Representative blots between young and old individuals. PBMCs were harvested from young $(\mathrm{A} ; n=10)$ and old $(\mathrm{B} ; n=10)$ unique cell donors and incubated at $37{ }^{\circ} \mathrm{C}$ for $24 \mathrm{~h}$. Cells were exposed to either control conditions dimethyl sulfoxide (DMSO), BAF (DMSO with $100 \mathrm{nM}$ BAF), or simulated starvation conditions (Rapa with 100 $\mathrm{nM} \mathrm{BAF}$ ) for $2 \mathrm{~h}$. Cells were harvested and prepared for Western blot analysis to measure the relative expression of LC3, HSP70, p62, and $\beta$-actin (an internal loading control)
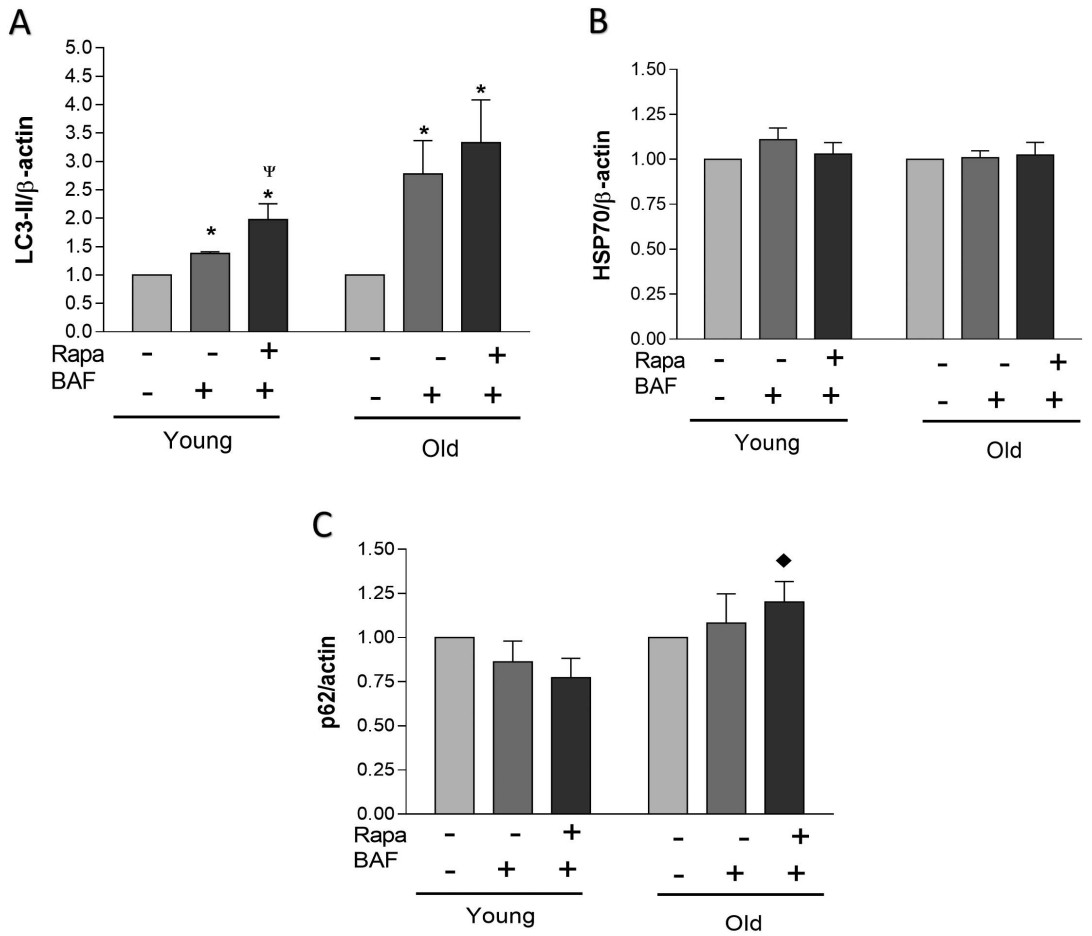

Fig. 2. Protein responses between young and old individuals. Densitometric values (A, B, and C) were obtained using Photoshop software and normalized to $\beta$-actin and set to 1 for control condition. Data represent mean \pm SEM (A, B, and $\mathrm{C}) . *$ indicates statistical significance $(p<0.05)$ within the respective group compared to control condition. $\Psi$ indicates statistical significance $(p<0.05)$ when compared to BAF condition within the respective group.

$\checkmark$ indicates statistical significance $(p<0.05)$ compared to the respective young group's treatment 
autophagy was possibly blunted in the older group in response to Rapa treatment when compared to the younger group (Figs 1 and 2). No statistical differences were observed when examining SQSTM1/p62 protein expression under any condition for either young or old groups; however, the older group elicited significantly higher SQSTM1/p62 expression under the Rapa treatment condition when compared to young. This may further suggest a reduced autophagic response in older individuals when compared to young, as SQSTM1/p62 protein accumulation is indicative of autophagy inhibition when treated with BAF. No significant Rapa-induced response was observed in HSP70 protein expression in younger or older individuals.

\section{Transcriptional regulation of autophagy and HSP70 in young and old individuals}

To further investigate the mechanisms involved in the autophagic response in older individuals, mRNA expression was examined for MAP1LC3B, SQSTM1/p62, and HSP70 (HSPA1A) (Fig. 3). The present findings indicate a significant increase in both MAP1LC3B and HSPA1A
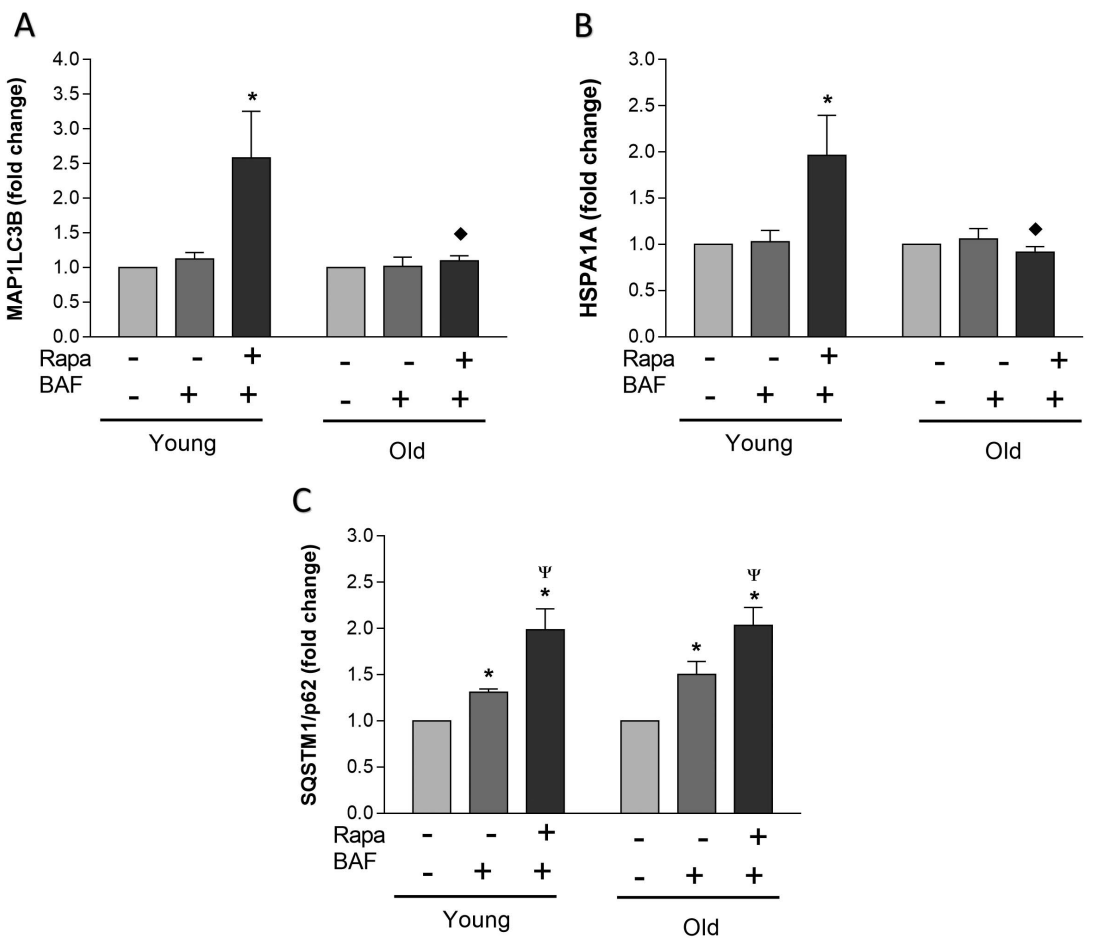

Fig. 3. Differences in gene expression between young and old individuals. PBMCs were harvested from young $(n=10)$ and old $(n=10)$ unique cell donors and incubated at $37{ }^{\circ} \mathrm{C}$ for $24 \mathrm{~h}$. Cells were exposed to either control conditions (DMSO vehicle), BAF (DMSO vehicle with $100 \mathrm{nM} \mathrm{BAF}$ ), or simulated starvation conditions (Rapa with $100 \mathrm{nM} \mathrm{BAF}$ ) for $2 \mathrm{~h}$. Cells were harvested and prepared for quantitative reverse transcription-polymerase chain reaction (qRT-PCR). The mRNA levels for MAP1LC3B (A), HSPA1A (B), and SQSTM1/p62 (C) were normalized to the reference gene $\beta$-actin and are represented as a fold change relative to the amount of $\beta$-actin. Data represent mean \pm SEM. * indicates statistical significance $(p<0.05)$ within the respective group compared to control condition. $\Psi$ indicates statistical significance $(p<0.05)$ when compared to BAF condition within the respective group. $\downarrow$ indicates statistical significance $(p<0.05)$ compared to the respective young group's treatment 
mRNA expression under Rapa treatment in younger individuals, whereas no significant increase in either MAP1LC3B or HSPA1A expression were observed in the older group. A significant fold increase was observed in SQSTM1/p62 mRNA expression in both younger and older groups in response to Rapa treatment. This indicates no transcriptional inhibition of the p62 in younger or older groups. An increase in HSPA1A mRNA in young and blunted response in old may indicate a transcriptional failure of the HSP70 system in the elderly.

\section{Discussion}

The autophagic and heat shock systems represent important cellular responses that enable cells to survive stressful conditions and restore cellular homeostasis (7, 30). Despite this observed elevation in basal autophagy, we demonstrate that the Rapa-induced autophagic response is suppressed in older individuals. This autophagic response in older individuals was associated with a decreased response in MAP1LC3B mRNA levels, but not the SQSTM1/p62 mRNA levels that were elevated in either group. Moreover, Rapa and BAF treatment resulted in an increased HSPA1A mRNA levels in young individuals, despite similar protein expression in either group.

\section{Basal autophagy in young versus old individuals}

While macroautophagy has previously been considered, an inducible type of autophagy, basal macroautophagy, has been reported to be essential for the maintenance of cellular homeostasis $(18,36)$. Indeed, increased basal autophagy activation may be beneficial initially, maintained activation could result in a malfunction of essential autophagic components and failure to upregulate this pathway when needed (33). This is evidenced by elevated levels of basal SQSTM1/p62 protein in aged individuals, indicating a lack of ubiquitinated substrates being transported to the autophagosome. Failure of this process may result in the aggregation of damaged molecules related to many age-associated diseases.

\section{Autophagic response to acute cellular stress in young versus old individuals}

Our findings indicate decreased autophagic activation in the presence of Rapa treatment in aged individuals when compared to their younger counterparts. This is supported by a significant elevation in LC3-II protein expression in younger individuals when compared to basal levels that is not observed in older individuals. Furthermore, in response to Rapa and BAF treatment, SQSTM1/p62 protein expression was significantly elevated in older individuals when compared to younger, suggesting a diminished autophagic response in aged individuals. An inability of the autophagic response in the presence of cellular stress results in inadequate turnover of organelles, such as mitochondria $(19,27)$. Accumulation of dysfunctional mitochondria has been reported to contribute to cellular aging through the release of free radicals, resulting in further protein damage within the cell $(19,27)$. In addition, while not measured in the present investigation, a repression of the autophagic response in older individuals may partially be due to changes in circulating levels of insulin and glucagon with age $(12,13)$. During periods of starvation, it has been proposed that younger organisms upregulate autophagy due to increases in glucagon levels, while insulin enhances the mTORmediated repression of autophagy (14). Characteristic of cellular aging is a gradual resistance to insulin, which in part may describe the observed basal activation of the autophagic response in older individuals $(9,16)$. 
Transcriptional regulation of autophagy in young versus old individuals

Our gene expression data suggest a failure of MAP1LC3B gene transcription in older individuals when compared to younger that is not reflected in the SQSTM1/p62 gene following Rapa treatment, suggesting an age-associated transcriptional blunting of autophagy. Rapa directly inhibits mTOR activation normally resulting in autophagy activation; however, suppression of LC3-II protein expression as observed in older individuals may be the result of an autophagic malfunction at the transcriptional level $(22,37)$. Induction of autophagy is typically accompanied by an increase in MAP1LC3B mRNA, as supported by the present findings in younger but not older individuals in response to cellular stress $(22,37)$. As LC3 protein turnover is increased during the periods of extensive cellular stress, it must be replenished through transcriptional induction of MAP1LC3B mRNA as demonstrated by others $(24,40)$. Thus, suppression of MAP1LC3B mRNA in response to Rapa treatment in older individuals, accompanied by a diminished response in LC3-II protein expression, may be indicative of a transcriptional malfunctioning of the autophagic response. There are no observed differences in the transcriptional response of SQSTM1/p62 between older and younger individuals, further emphasizing a failure to synthesize LC3-II as a primary contributor to the observed age-associated inhibition of autophagy.

\section{Interconnection of heat shock response and autophagy}

The heat shock response represents a protein management system in which HSPs prevent protein aggregation and facilitate refolding (32). Under stressful conditions, increased HSP70 protein expression allows cells to survive harsh conditions and restore cellular homeostasis through maintenance of proper protein structure $(7,30)$. In response to $2 \mathrm{~h}$ of Rapa and BAF treatment, we found no change in HSP70 protein expression in either younger or older individuals; however, a significant upregulation of HSPA1A mRNA expression was observed in younger individuals but not observed in older. It has previously been reported that Rapa has minimal impact on the HSP70 mRNA translation pathway in Drosophila, thus observed HSPA1A levels may represent cell-specific differences in response to elevated autophagy induction (15). Autophagy functioning to maintain energy and protein homeostasis has been shown to be under the regulatory control of HSP70 (11). Our findings are in agreement with past research, indicating minimal acute HSP70 protein expression following autophagy induction in response to Rapa treatment (11). Upregulation of HSPA1A mRNA in young following Rapa treatment may be indicative of an initial heat shock response; however, as HSP70 and autophagy were not measured beyond $2 \mathrm{~h}$ following Rapa treatment, this remains purely speculative. Furthermore, lack of HSPA1A mRNA response in the older group may contribute to the observed autophagic malfunctioning on a transcriptional level. Further research is necessary to fully understand the mechanism of HSP70 regulation on autophagy in aged individuals.

\section{Conclusions}

In summary, we found that the autophagic response is suppressed in older individuals following Rapa and BAF treatment when compared to younger individuals. The lack of change in the older group for MAP1LC3B mRNA expression may be indicative of malfunctioning transcriptional regulation of autophagy in this population. It should be noted that these findings are tissue-specific to PBMCs in younger and older individuals. Further 
research is necessary to understand the mechanisms of this observed suppression of the autophagic and heat shock systems in the elderly in other tissues.

\section{REFERENCES}

1. Alevy YG, Patel AC, Romero AG, Patel DA, Tucker J, Roswit WT, Miller CA, Heier RF, Byers DE, Brett TJ, Holtzman MJ: IL-13-induced airway mucus production is attenuated by MAPK13 inhibition. J. Clin. Invest. 122, 4555-4568 (2012)

2. Alvers AL, Wood MS, Hu D, Kaywell AC, Dunn WA, Jr, Aris JP: Autophagy is required for extension of yeast chronological life span by rapamycin. Autophagy 5, 847-849 (2009)

3. Bjedov I, Toivonen JM, Kerr F, Slack C, Jacobson J, Foley A, Partridge L: Mechanisms of life span extension by rapamycin in the fruit fly Drosophila melanogaster. Cell Metab. 11, 35-46 (2010)

4. Canto C, Jiang LQ, Deshmukh AS, Mataki C, Coste A, Lagouge M, Zierath JR, Auwerx J: Interdependence of AMPK and SIRT1 for metabolic adaptation to fasting and exercise in skeletal muscle. Cell Metab. 11, 213-219 (2010)

5. Coffey EE, Beckel JM, Laties AM, Mitchell CH: Lysosomal alkalization and dysfunction in human fibroblasts with the Alzheimer's disease-linked presenilin 1 A246E mutation can be reversed with cAMP. Neuroscience 263, 111-124 (2014)

6. Colman RJ, Anderson RM, Johnson SC, Kastman EK, Kosmatka KJ, Beasley TM, Allison DB, Cruzen C, Simmons HA, Kemnitz JW, Weindruch R: Caloric restriction delays disease onset and mortality in rhesus monkeys. Science 325, 201-204 (2009)

7. Craig EA, Weissman JS, Horwich AL: Heat shock proteins and molecular chaperones: mediators of protein conformation and turnover in the cell. Cell 78, 365-372 (1994)

8. Cuervo AM: Autophagy: many paths to the same end. Mol. Cell. Biochem. 263, 55-72 (2004)

9. Cuervo AM: Autophagy and aging: keeping that old broom working. Trends Genet. 24, 604-612 (2008)

10. Dokladny K, Myers OB, Moseley PL: Heat shock response and autophagy - cooperation and control. Autophagy $11,200-13(2015)$

11. Dokladny K, Zuhl MN, Mandell M, Bhattacharya D, Schneider S, Deretic V, Moseley PL: Regulatory coordination between two major intracellular homeostatic systems: heat shock response and autophagy. J. Biol. Chem. 288, 14959-14972 (2013)

12. Donati A, Cavallini G, Paradiso C, Vittorini S, Pollera M, Gori Z, Bergamini E: Age-related changes in the regulation of autophagic proteolysis in rat isolated hepatocytes. J. Gerontol. A Biol. Sci. Med. Sci. 56, B288-B293 (2001)

13. Donati A, Recchia G, Cavallini G, Bergamini E: Effect of aging and anti-aging caloric restriction on the endocrine regulation of rat liver autophagy. J. Gerontol. A Biol. Sci. Med. Sci. 63, 550-555 (2008)

14. Droge W, Kinscherf R: Aberrant insulin receptor signaling and amino acid homeostasis as a major cause of oxidative stress in aging. Antioxid. Redox Signal. 10, 661-678 (2008)

15. Duncan RF: Rapamycin conditionally inhibits Hsp90 but not Hsp70 mRNA translation in Drosophila: implications for the mechanisms of Hsp mRNA translation. Cell Stress Chaperones 13, 143-155 (2008)

16. Fink RI, Kolterman OG, Griffin J, Olefsky JM: Mechanisms of insulin resistance in aging. J. Clin. Invest. 71, 1523-1535 (1983)

17. Hansen M, Chandra A, Mitic LL, Onken B, Driscoll M, Kenyon C: A role for autophagy in the extension of lifespan by dietary restriction in C. elegans. PLoS Genet. 4, e24 (2008)

18. Hara T, Nakamura K, Matsui M, Yamamoto A, Nakahara Y, Suzuki-Migishima R, Yokoyama M, Mishima K, Saito I, Okano H, Mizushima N: Suppression of basal autophagy in neural cells causes neurodegenerative disease in mice. Nature 441, 885-889 (2006)

19. Jin S: Autophagy, mitochondrial quality control, and oncogenesis. Autophagy 2, 80-84 (2006)

20. Kabeya Y, Mizushima N, Ueno T, Yamamoto A, Kirisako T, Noda T, Kominami E, Ohsumi Y, Yoshimori T: LC3, a mammalian homologue of yeast Apg8p, is localized in autophagosome membranes after processing. EMBO J. 19, 5720-5728 (2000)

21. Kenyon CJ: The genetics of ageing. Nature 464, 504-512 (2010)

22. Kirisako T, Baba M, Ishihara N, Miyazawa K, Ohsumi M, Yoshimori T, Noda T, Ohsumi Y: Formation process of autophagosome is traced with Apg8/Aut7p in yeast. J. Cell Biol. 147, 435-446 (1999) 
23. Klionsky DJ, Abdelmohsen K, Abe A, Abedin MJ, Abeliovich H, Acevedo Arozena A..., Zughaier SM: Guidelines for the use and interpretation of assays for monitoring autophagy (3rd edition). Autophagy 12, 1-222 (2016)

24. Kouroku Y, Fujita E, Tanida I, Ueno T, Isoai A, Kumagai H, Ogawa S, Kaufman RJ, Kominami E, Momoi T: ER stress (PERK/eIF2alpha phosphorylation) mediates the polyglutamine-induced LC3 conversion, an essential step for autophagy formation. Cell Death Differ. 14, 230-239 (2007)

25. Lapierre LR, Kumsta C, Sandri M, Ballabio A, Hansen M: Transcriptional and epigenetic regulation of autophagy in aging. Autophagy 11, 867-880 (2015)

26. Lee JH, Budanov AV, Park EJ, Birse R, Kim TE, Perkins GA, Ocorr K, Ellisman MH, Bodmer R, Bier E, Karin M: Sestrin as a feedback inhibitor of TOR that prevents age-related pathologies. Science 327, 1223-1228 (2010)

27. Lemasters JJ: Selective mitochondrial autophagy, or mitophagy, as a targeted defense against oxidative stress, mitochondrial dysfunction, and aging. Rejuvenation Res. 8, 3-5 (2005)

28. Levine B, Klionsky DJ: Development by self-digestion: molecular mechanisms and biological functions of autophagy. Dev. Cell 6, 463-477 (2004)

29. Levine B, Kroemer G: Autophagy in the pathogenesis of disease. Cell 132, 27-42 (2008)

30. Liang P, MacRae TH: Molecular chaperones and the cytoskeleton. J. Cell Sci. 110, 1431-1440 (1997)

31. Lipinski MM, Zheng B, Lu T, Yan Z, Py BF, Ng A, Xavier RJ, Li C, Yankner BA, Scherzer CR, Yuan J: Genome-wide analysis reveals mechanisms modulating autophagy in normal brain aging and in Alzheimer's disease. Proc. Natl. Acad. Sci. U. S. A. 107, 14164-14169 (2010)

32. Mayer MP, Bukau B: Hsp70 chaperones: cellular functions and molecular mechanism. Cell. Mol. Life Sci. 62, 670-684 (2005)

33. Meijer AJ, Codogno P: Macroautophagy: protector in the diabetes drama? Autophagy 3, 523-526 (2007)

34. Melendez A, Talloczy Z, Seaman M, Eskelinen EL, Hall DH, Levine B: Autophagy genes are essential for dauer development and life-span extension in C. elegans. Science 301, 1387-1391 (2003)

35. Mizushima N, Yoshimori T, Levine B: Methods in mammalian autophagy research. Cell 140, 313-326 (2010)

36. Nakai A, Yamaguchi O, Takeda T, Higuchi Y, Hikoso S, Taniike M, Omiya S, Mizote I, Matsumura Y, Asahi M, Nishida K, Hori M, Mizushima N, Otsu K: The role of autophagy in cardiomyocytes in the basal state and in response to hemodynamic stress. Nat. Med. 13, 619-624 (2007)

37. Nara A, Mizushima N, Yamamoto A, Kabeya Y, Ohsumi Y, Yoshimori T: SKD1 AAA ATPase-dependent endosomal transport is involved in autolysosome formation. Cell Struct. Funct. 27, 29-37 (2002)

38. Ohsumi Y, Mizushima N: Two ubiquitin-like conjugation systems essential for autophagy. Semin. Cell Dev. Biol. 15, 231-236 (2004)

39. Ploumi C, Daskalaki I, Tavernarakis N: Mitochondrial biogenesis and clearance: a balancing act. FEBS J. 284, 183-195 (2017)

40. Rouschop KM, van den Beucken T, Dubois L, Niessen H, Bussink J, Savelkouls K, Keulers T, Mujcic H, Landuyt W, Voncken JW, Lambin P, van der Kogel AJ, Koritzinsky M, Wouters BG: The unfolded protein response protects human tumor cells during hypoxia through regulation of the autophagy genes MAP1LC3B and ATG5. J. Clin. Invest. 120, 127-141 (2010)

41. Rubinsztein DC, Marino G, Kroemer G: Autophagy and aging. Cell 146, 682-695 (2011)

42. Simonsen A, Cumming RC, Brech A, Isakson P, Schubert DR, Finley KD: Promoting basal levels of autophagy in the nervous system enhances longevity and oxidant resistance in adult Drosophila. Autophagy 4, 176-184 (2008)

43. Tan CC, Yu JT, Tan MS, Jiang T, Zhu XC, Tan L: Autophagy in aging and neurodegenerative diseases: implications for pathogenesis and therapy. Neurobiol. Aging 35, 941-957 (2014)

44. Toth ML, Sigmond T, Borsos E, Barna J, Erdelyi P, Takacs-Vellai K, Orosz L, Kovacs AL, Csikos G, Sass M, Vellai T: Longevity pathways converge on autophagy genes to regulate life span in Caenorhabditis elegans. Autophagy 4, 330-338 (2008)

45. Yamamoto A, Tagawa Y, Yoshimori T, Moriyama Y, Masaki R, Tashiro Y: Bafilomycin A1 prevents maturation of autophagic vacuoles by inhibiting fusion between autophagosomes and lysosomes in rat hepatoma cell line, H-4-II-E cells. Cell Struct. Funct. 23, 33-42 (1998) 